OPEN ACCESS

Edited by:

Takao Namaiki,

Chiba University, Japan

Reviewed by:

Akio Inui,

Kagoshima University, Japan Yasuhito Uezono,

Jikei University School of

Medicine, Japan

*Correspondence:

Chihiro Yamada

yamada_chihiro@mail.tsumura.co.jp

Specialty section:

This article was submitted to

Clinical Nutrition,

a section of the journal

Frontiers in Nutrition

Received: 20 August 2021 Accepted: 09 November 2021

Published: 09 December 2021

Citation:

Yamada C, Hattori T, Ohnishi S and Takeda H (2021) Ghrelin Enhancer, the

Latest Evidence of Rikkunshito.

Front. Nutr. 8:761631.

doi: 10.3389/fnut.2021.761631

\section{Ghrelin Enhancer, the Latest Evidence of Rikkunshito}

\author{
Chihiro Yamada ${ }^{1 *}$, Tomohisa Hattori ${ }^{1}$, Shunsuke Ohnishi ${ }^{2}$ and Hiroshi Takeda ${ }^{3,4}$ \\ ${ }^{1}$ Tsumura Kampo Research Laboratories, Tsumura \& Co., Ibaraki, Japan, ${ }^{2}$ Laboratory of Molecular and Cellular Medicine, \\ Faculty of Pharmaceutical Sciences, Hokkaido University, Sapporo, Japan, ${ }^{3}$ Gastroenterology, Tokeidai Memorial Hospital, \\ Sapporo, Japan, ${ }^{4}$ Faculty of Pharmaceutical Sciences, Hokkaido University, Sapporo, Japan
}

Rikkunshito is a Japanese herbal medicine (Kampo) that has been attracting attention and researched by many researchers not only in Japan but also worldwide. There are 214 rikkunshito articles that can be searched on PubMed by August 2021. The reason why rikkunshito has attracted so much attention is due to an epoch-making report (Gastroenterology, 2008) discovered that rikkunshito promotes the secretion of the orexigenic peptide ghrelin. Since then, many researchers have discovered that rikkunshito has a direct effect on the ghrelin receptor, GHS-R1a, and an effect of enhancing the ghrelin signal to the brain. Additionally, a lot of evidence that rikkunshito is expected to be effective for various gastrointestinal diseases have also been demonstrated. Numerous basic and clinical studies have suggested that rikkunshito affects (i) various discomforts caused by anticancer drugs, gastroesophageal reflux disease, functional dyspepsia, (ii) various stress-induced anorexia, (iii) hypophagia in the elderly, and (iv) healthy lifespan. In this review, as one who discovered the ghrelin enhancer effect of rikkunshito, we will review the research of rikkunshito so far and report on the latest research results.

Keywords: ghrelin, GHS-R, anorexia, rikkunshito, Kampo, stress, aging

\section{RIKKUNSHITO}

Rikkunshito is one of the prescriptions described in the old medical book Return of Spring from All Kinds of Diseases compiled by Kyoenken in 1587. Rikkunshito comprises eight herbal medicines, Atractylodis lanceae rhizoma, Ginseng radix, Pinelliae tuber, Hoelen, Zizyphi fructus, Aurantii nobilis pericarpium, Glycyrrhizae radix and Zingiberis rhizoma. It was used for patients with gastrointestinal weakness, loss of appetite, epigastrium, tiredness, anemia, and chills in the limbs. In Japan, it is an insurance coverage drug for gastritis, gastric atony, gastroptosis, indigestion, loss of appetite, stomach pain, and vomiting, which is covered by a doctor's prescription. The first high-quality evidence is a multicenter comparative study of TJ-43 rikkunshito for indefinite gastrointestinal complaints, such as chronic gastritis by Harasawa et al. (1). Additionally, a multicenter, double-blind study using rikkunshito has recently been conducted, including proton pump inhibitor refractory non-erosive reflux disease (NERD, $n=242$ ) (2) and functional dyspepsia $(n=192)$ (3), and it was proven to be effective for them. Rikkunshito was compared to the placebo treatment group, and the degree of improvement of total and the acid-related dysmotility symptom scores of the frequency scale for the symptoms of gastroesophageal reflux disease (FSSG) after the 8-week treatment was significantly greater in the rikkunshito group than in the placebo group. Rikkunshito also significantly increased the global assessment of overall treatment efficacy in functional dyspepsia patients and improved upper gastrointestinal symptoms after 8 weeks, 
especially postprandial fullness/early satiety and bloating. Thus, except acid secretion inhibitors and acotiamide, there is poor evidence of drugs for digestive system complaints so far, so the accumulation of clear evidence by Japanese Kampo medicine is an important event.

Additionally, rikkunshito's mechanisms of action have been extensively studied in detail. Rikkunshito has been proven to enhance gastric emptying (4-6) and promote adaptive relaxation reaction (6-10) of the stomach from basic and clinical aspects. These actions are not merely single pharmacological actions, such as increased gastric motility or decreased gastric acid secretion, but have the characteristic of enhancing the overall function of the stomach. Thus, it can be said that the mechanism of action of rikkunshito is significantly different from that of new drugs with a single pharmacological action.

Furthermore, the epoch-making evidence that rikkunshito was made known to global gastroenterologists was believed to be discovering the ghrelin-enhancing effect of the orexigenic peptide by rikkunshito (11). Inspired by the common characteristics of patients after taking rikkunshito, we discovered that rikkunshito may enhance the action of ghrelin and proved the first evidence. After that, many researchers proceeded with further detailed research, and rikkunshito enhanced the gene expression of the ghrelin receptor GHS-R1a (12) and that the signal transduction of ghrelin was enhanced by improving the binding between ghrelin and GHS-R1a (13).

This review focuses on the effects of rikkunshito on ghrelin and describes the latest evidence and the following possibilities regarding the potential treatment of rikkunshito for various diseases.

\section{ACTION AND MECHANISM ON GHRELIN What Is Ghrelin?}

Ghrelin is a unique peptide with 28 amino acids and an n-octanoyl group. Its main production site is localized to $\mathrm{X} / \mathrm{A}$-like cells in the gastric mucosa (14). The hunger signal from periphery is increased by the production of ghrelin, for example, hypoglycemia. As a reflection, it is also increased in the blood. The orexigenic activity of ghrelin is triggered by the serine 3 -acyl modification of octanoic acid (acylated ghrelin). This acylation process is catalyzed by the gastric membrane-binding protein, ghrelin $\mathrm{O}$-acyltransferase (GOAT) (15). However, acylated ghrelin released into tissues or blood is immediately metabolized by liver-derived butyrylcholinesterase (16) to des-acyl ghrelin, which has no orexigenic effect (Figure 1). A difficult point in measuring the active form of acylated ghrelin in clinical research is the instability of acylated ghrelin. The half-life after intravenous injection of acylated ghrelin is $8 \mathrm{~min}$ in rats (17). In the case of animal experiments, it should be conducted within 3-5 min from blood sampling to centrifugation by multiple researchers before adding concentrated hydrochloric acid and freezing in liquid nitrogen. A deep freezer at $-80^{\circ} \mathrm{C}$ is optimal for storage. However, it is not easy to freeze within this time after collecting blood from a patient. Therefore, if the conditions at the time of blood collection are different, it is expected that the acylated ghrelin value will also differ significantly from facility to facility or from day to day.

The target of the ghrelin ligand is growth hormone secretagogue receptor 1a (GHS-R1a), a 7-transmembrane G protein-coupled receptor composed of 366 amino acid residues. GHS-R1a is present in the terminal of the vagal afferent nerve, pancreatic cells, spleen, myocardium, bone, fat, thyroid gland, adrenal gland, and immune cells $(18,19)$. Particularly, it is also densely expressed in the hypothalamic nucleus in the central nervous system $(18,19)$. The peripheral to the central ghrelin signal is initiated by the binding of GHS-R1a present at the vagal nerve terminal in the gastric mucosa to acylated ghrelin, and the signal to the central nervous system is transmitted. Ghrelin bound to GHS-R1a transmits its starvation signal via the solitary nucleus (NTS) of the medulla oblongata to neuropeptide Y (NPY)/Agouti-related peptide (AgRP) neurons, which are localized in the arcuate nucleus of the hypothalamus and the signal is transmitted to higher centers. Ghrelin stimulates NPY/AgRP neurons and promotes the production of NPY/AgRP peptides and proceeds to induce appetite (20) and promotes gastrointestinal fasting contraction via the vagus nerve efferent pathway $(21,22)$. GHS-R1a is also localized in the ventral tegmental area (23) and hippocampus (24). Thus, in addition to the action on appetite and energy metabolism, ghrelin may also be involved in cognition and memory and is thought to play an essential role in maintaining neuropsychiatric homeostasis in both peripheral and central tissues.

Serotonin and the adrenaline system play a major role in energy metabolism and regulate the secretion of ghrelinproducing cells, X/A-like cells in the gastric mucosa (11, 2527). Particularly, intraperitoneal (IP) administration of an agonist of serotonin $2 \mathrm{~B}$ receptor $\left(5-\mathrm{HT}_{2 \mathrm{~B}} \mathrm{R}\right) \mathrm{BW} 723 \mathrm{C} 86$ or an agonist of $5-\mathrm{HT}_{2 \mathrm{C}} \mathrm{R}$, meta-chlorophenyl piperazine (mCPP), to rats significantly reduces acylated ghrelin levels in peripheral blood $(11,25)$. Administration of the $5-\mathrm{HT}_{2 \mathrm{~B}} \mathrm{R}$ or ${ }_{2 \mathrm{C}} \mathrm{R}$ antagonists significantly reversed decreases in peripheral ghrelin and food intake in several disease models (11, 28-30). Cell damage releases large amounts of serotonin after chemotherapy such as cisplatin administration, but it is easy to imagine activating $5-\mathrm{HT}_{2 \mathrm{~B}} \mathrm{R}$ and $5-\mathrm{HT}_{2 \mathrm{C}} \mathrm{R}$ present in the gastric smooth muscle and brain. Administering these receptor antagonists to cisplatin-treated rats simultaneously restores reduced food intake and peripheral acylated ghrelin (11).

Acute stress increases the secretion of adrenaline and activates various receptors. Clonidine, an adrenergic $\alpha 2$ receptor agonist, also lowered peripheral acylated ghrelin concentration. Conversely, the addition of noradrenaline or adrenaline to the ghrelinoma cell lines stimulated ghrelin secretion, and this effect was blocked by atenolol (31), a selective $\beta 1$ adrenergic antagonist. Systemic administration of isoproterenol and denopamine restores reduced blood acylated ghrelin levels in stress models. In a stress-like model with intracerebroventricular (ICV) urocortin injection, the administration of adrenergic $\alpha$ receptor antagonist phentolamine and the $\alpha 2$ receptor antagonist yohimbine significantly improved the decreased acylated levels (32). The effect disappeared when administered along with a ghrelin receptor antagonist. This means that adrenergic $\alpha 2$ 


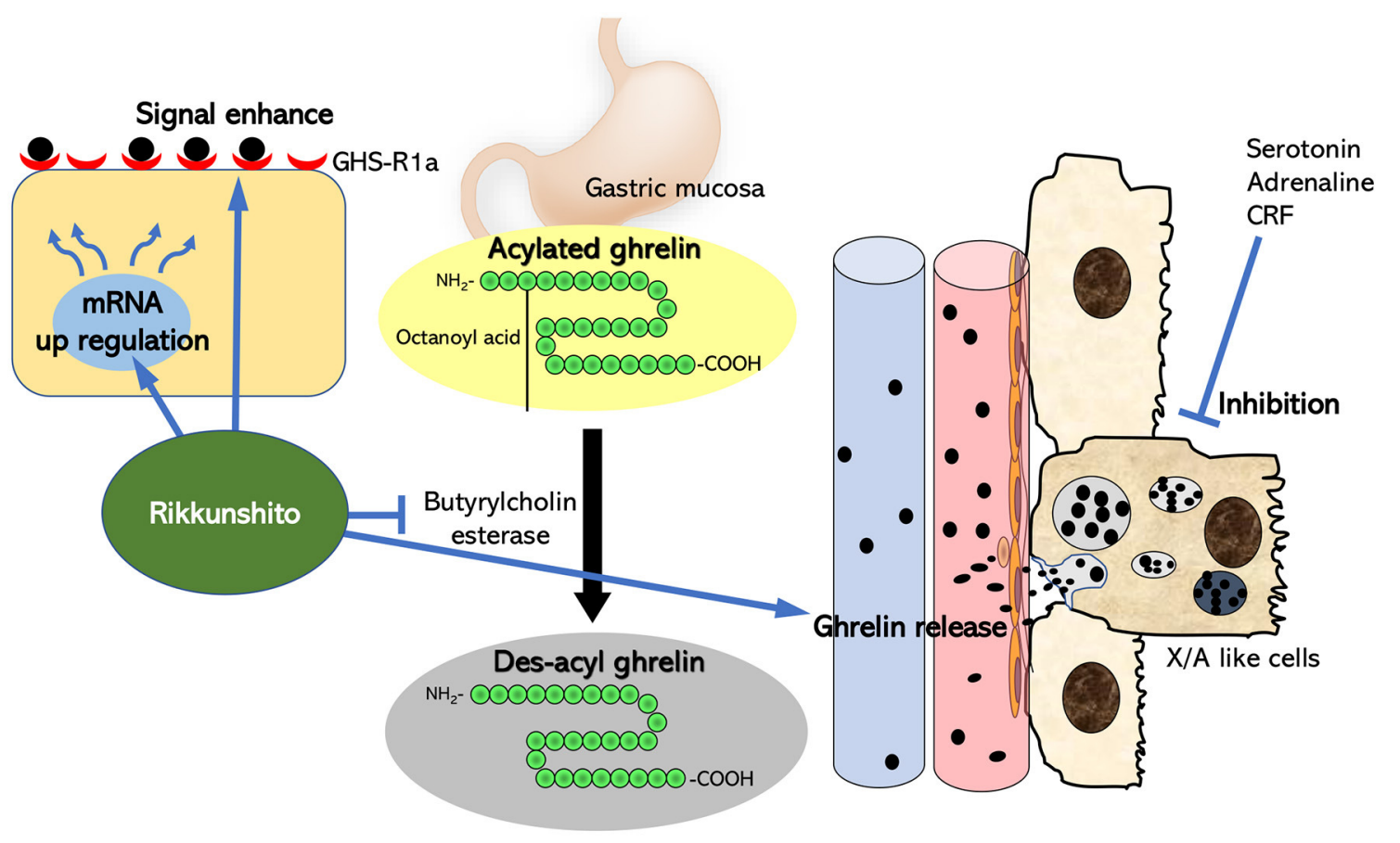

FIGURE 1 | Summary of the mechanism of action of rikkunshito as a ghrelin enhancer. Ghrelin is produced from X/A-like cells near the gastric mucosa, and the ghrelin receptors at the ends of the vagus nerve in the surrounding tissues are stimulated. Most of it migrates to blood vessels and is carried to the brain. Serotonin, adrenaline, and CRF negatively regulate ghrelin secretion in the periphery. Rikkunshito suppresses the decrease in ghrelin that occurs in diseases by antagonistically acting on these factors with receptors in the periphery or central nervous system. Furthermore, rikkunshito enhances the binding of ghrelin to the ghrelin receptor and also acts on the ghrelin signal transduction system itself to enhance the ghrelin signal.

receptor stimulation negatively regulated feeding through a decrease in ghrelin receptor activation. ICV administration of the corticotropin-releasing factor (CRF), a trigger factor for the HPA axis during stress, also reduces peripheral blood acylated ghrelin levels and food intake (29). Thus, abnormal acylated ghrelin secretion may be noticed in diseases in which these factors are closely related to pathology.

\section{Promotion of Ghrelin Secretion by Rikkunshito}

\section{Normal Animals and Healthy Volunteers}

A few reports examine the effects of the administration of rikkunshito on normal mice to increase appetite and acylated ghrelin production. Matsumura et al. measured blood acylated ghrelin concentration and gastric gene expression at $0.7-1.4 \%$ drinking water administration of rikkunshito to normal mice and showed a significant increase in the rikkunshito group compared with the saline-treated group (33). However, in our preliminary study, the amount of acylated ghrelin in the blood tended to increase $180 \mathrm{~min}$ after the administration of rikkunshito $1 \mathrm{~g} / \mathrm{kg}$ by gavage (distilled water administration group, $55.7 \pm 9.2 \mathrm{fmol} / \mathrm{mL}$, vs. rikkunshito administration group, $72.3 \pm 5.9 \mathrm{fmol} / \mathrm{mL}$ ), and des-acyl concentration decreased (distilled water administration group, $729.6 \pm 74.1 \mathrm{fmol} / \mathrm{mL}$, vs. rikkunshito administration group, $407.1 \pm 30 \mathrm{fmol} / \mathrm{mL}$ ). It is considered that the difference in this result depends on the difference in administration period and administration method. Additionally, Matsumura et al. demonstrated that administration of rikkunshito $7.5 \mathrm{~g} /$ day to 21 healthy volunteers for 2 weeks significantly increased blood acylated ghrelin levels compared to that before administration (33). It is considered that the maintenance of physiological homeostasis related to the secretion of appetite-related hormones is strictly controlled. Therefore, the effect of a single dose of rikkunshito on healthy subjects may be limited. To affect normal ghrelin secretion function, rikkunshito may require continuous administration.

\section{Side Effects of Chemotherapy and Cancer Cachexia \\ Animal Model}

Table 1 summarizes the major indexes and ghrelin-promoting effects of rikkunshito on anticancer drug administration, organ removal, and cancer cachexia. Administering cisplatin to rodents results in reduced food intake. Peritoneal administration of cisplatin at $2 \mathrm{mg} / \mathrm{kg}$ significantly reduces rat feeding under fasting and free fed conditions up to $24 \mathrm{~h}$. Gavage oral administration at a dose of $1 \mathrm{~g} / \mathrm{kg}$ of rikkunshito was observed to significantly suppress the reduction in food intake due to cisplatin administration compared to saline-administered rats (11-13, 34, 35). Intraperitoneal administration of cisplatin 2 $\mathrm{mg} / \mathrm{kg}$ decreases food intake and decreases blood acylated ghrelin levels and hypothalamic acylated ghrelin release $2 \mathrm{~h}$ 
after administration. Rikkunshito has been proven to abolish these declines $(11,12,35)$. Similarly, rikkunshito suppressed the decrease in food intake in rats given intraperitoneally at a higher dose of cisplatin (6 mg/kg) (36). Thus, the effect of rikkunshito on ghrelin is very reproducible, and it has been proven that rikkunshito inhibits feeding reduction and an acylated ghrelin secretion-promoting effect in cisplatin-administered rats. Moreover, in cancer-bearing animals, experimental exposure to cancer cells induces cachexia, depending on the type of cancer and the duration of the experiment, resulting in a marked decrease in food intake and body weight. Additionally, a significant reduction in food intake and body weight is also observed in gastrectomized rats (37). An extreme decline in feeding leads to increased starvation and induction of signal abnormalities in peripheral appetite-promoting peptides, despite the lack of appetite in these animal models. Particularly, peripheral acylated ghrelin increases, and exogenous acylated ghrelin reactivity also decreases the so-called ghrelin resistance $(13,38)$. Rikkunshito administration to cancer cachexia rats is shown to improve the decrease in food intake and prolong life. However, rikkunshito did not further increase blood acylated ghrelin. Rikkunshito not only increases peripheral ghrelin but also stimulates ghrelin receptor signaling and stimulates feeding $(13,38)$. This mechanism of action of rikkunshito will be focused on in detail at the bottom.

\section{Clinical Study}

In gastric cancer patients (39-41) and uterine cervical or corpus cancer patients (42), the efficacy of rikkunshito for ghrelin concentration and gastrointestinal dysfunction, including feeding, was evaluated. Ohno et al. reported that rikkunshito at a dose of $7.5 \mathrm{~g}$ /day suppressed the increase in oral intake and the decrease in acylated ghrelin due to cisplatin administration from the start of the combined administration of S-1 and cisplatin to patients with gastric cancer (39). Similarly, Takiguchi et al. (40) observed an increase in the acylated ghrelin ratio to total ghrelin at 4 weeks after administration of rikkunshito and an improvement in the Dysfunction after Upper Gastrointestinal Surgery for Cancer (DAUGS) and visual analog scale. These results clearly suggest that rikkunshito clinically promoted ghrelin secretion and suppressed feedingrelated decline. However, in patients with proximal gastrectomy, weight gain and increase in the Gastrointestinal Symptom Rating Scale (GSRS) were noticed after administering rikkunshito. Still, they did not affect ghrelin levels (41). These findings suggest that rikkunshito improves gastrointestinal symptoms in patients with gastrectomy, but its effect on ghrelin has various results. This may be mediated by the fact that the main production site of ghrelin is the stomach. The conditions for collecting blood samples and the conditions for measuring acylated ghrelin may differ at each facility. Ohnishi et al. (42) found that rikkunshito (7.5 $\mathrm{g} /$ day) administration for 2 weeks in patients with cervical cancer improved appetite up to 2-6 days after paclitaxel administration and delayed onset $24-120 \mathrm{~h}$ later, and it significantly suppressed nausea and vomiting. However, acylated ghrelin did not change even after administration of an anticancer drug, and no effect was found by rikkunshito. Moreover, it was reported that there was no effect on nausea and vomiting by chemotherapy for lung cancer patients in the group taking rikkunshito $7.5 \mathrm{~g} /$ day for 7 days at the same time (43) but for anorexia. The 14-day administration of rikkunshito ( $7.5 \mathrm{~g} /$ day) inhibited the decrease in plasma acylated ghrelin, and the rate of decrease in calorie intake was lower in rikkunshito than in the control course (18 vs. $25 \%, P=$ 0.025) (44). Another researcher reported that the median rate of reduction in food intake was significantly lower with rikkunshito than without it (2 vs. $30 \% ; P=0.02$ ) (45). Median acylated ghrelin increased significantly from day 3 to day 8 in patients on both courses with and without rikkunshito $(9.6-15.7 \mathrm{fmol} / \mathrm{mL}, \mathrm{P}$ $<0.0001$; control, $10.2-17.8 \mathrm{fmol} / \mathrm{mL}, P=0.0002)$. The rate of median increase in plasma acylated ghrelin levels between days 3 and 8 tended to be higher in the rikkunshito than in the control course (68 vs. $48 \%, P=0.08$ ). For delayed gastric emptying after pancreaticoduodenectomy, the 21-day administration of rikkunshito showed a significantly upregulating in total ghrelin and acylated ghrelin levels compared to preoperative, but no obvious effect on delayed gastric emptying was observed (46). It is believed that the effectiveness of rikkunshito will become clearer in future large-scale studies.

\section{Stress-Induced Loss of Appetite}

Stress is closely associated with appetite and exhibits an entirely different phenotype depending on the quality and duration of stress. Acute stress and stress loads that have a strong impact may primarily suppress feeding (52). In addition, a combination with chronic and mild stress may increase appetite and alter food preferences $(53,54)$. Previously, it has been reported that abnormal dynamics in acylated ghrelin are observed with stress loading and mediate abnormality in appetite. This review describes the effect of rikkunshito on the stress-induced loss of appetite.

\section{Animal Model}

Blood adrenocorticotropic hormone (ACTH) and corticosterone on the HPA axis in the rodent model are used as indicators of the degree of an acute stress response. Previous studies have not investigated in detail whether rikkunshito inhibits the HPA axis. It was confirmed that the administration of rikkunshito to stressloaded aged mice significantly decreased the increase in ACTH or corticosterone value (55). This indicates that rikkunshito may act in a suppressive manner on stress itself.

Yakabi et al. $(32,47)$ and Harada (49) demonstrated that the ICV administration of urocortin 1 , which has a strong affinity for the CRF receptor, significantly reduces feeding behavior and abnormal movement of the upper gastrointestinal tract. The concentration of acylated ghrelin in the peripheral blood was significantly reduced, simultaneously, and the supplementation of acylated ghrelin to the urocortin-treated rat significantly improved this decrease in food intake. Urocortin-induced reduction of plasma ghrelin and food intake were restored by CRF2 receptor antagonist. Administration of rikkunshito to urocortin-treated rats significantly improved reduced food intake, abnormal gastrointestinal motility, and acylated ghrelin levels $(32,47,49)$. The adrenergic $\alpha 2$ receptor was activated in urocortin-administered rats, and it was also found that 
TABLE 1 | The effect of rikkunshito on the main evaluation and on ghrelin in each study.

\begin{tabular}{|c|c|c|c|c|}
\hline Years & Basic research & Ghrelin & Index & References \\
\hline 2008 & Cisplatin-treated rats & $\uparrow$ & Food intake $\uparrow$ & Takeda et al. (11) \\
\hline 2010 & Cisplatin-treated rats & $\uparrow$ & Food intake $\uparrow$ & Yakabi et al. (12) \\
\hline 2011 & Cancer cachexia rats & $\uparrow$ & Food intake, survival $\uparrow$ & Fujitsuka et al. (13) \\
\hline 2011 & Cisplatin-treated rats & $\uparrow$ & Food intake $\uparrow$ & Sadakane et al. (35) \\
\hline 2013 & Cisplatin-treated rats & - & Food intake $\uparrow$ & Yoshimura et al. (36) \\
\hline 2016 & Gastrectomied rats & $\rightarrow$ & Food intake $\uparrow$ & Taguchi et al. (37) \\
\hline 2017 & Gastric cancer rats & signal $\uparrow$ & Food intake $\uparrow$ & Terawaki et al. (38) \\
\hline Years & Clinical research & Ghrelin & Index & References \\
\hline 2011 & Cisplatin-treated patients with gastric cancer & $\nearrow$ & Food consumption $\uparrow$ & Ohno et al. (39) \\
\hline 2011 & Cancer cachexia patients & $\uparrow$ & Survival $\uparrow$ & Fujitsuka et al. (13) \\
\hline 2013 & Gastric cancer & $\uparrow$ & Food consumption, DAUGS score $\uparrow$ & Takiguchi et al. (40) \\
\hline 2013 & Gastrectomy & $\rightarrow$ & Body weight, GSRS $\uparrow$ & Gunji et al. (41) \\
\hline 2017 & Uterine cervical or corpus cancer patients & $\rightarrow$ & CINV $\downarrow$ & Ohnishi et al. (42) \\
\hline 2017 & Lung cancer patients with chemotherapy & - & $\mathrm{CINV} \rightarrow$ & Harada et al. (43) \\
\hline 2019 & Cisplatin-treated patients with esophageal cancer & $\uparrow$ & Food consumption $\uparrow$ & Hamai et al. (45) \\
\hline 2020 & Cisplatin-treated patients with lung cancer & $\uparrow$ & Food consumption $\uparrow$ & Yoshiya et al. (44) \\
\hline 2020 & Pancreaticoduodenectomy & $\rightarrow$ & Delayed gastric emptying $\rightarrow$ & Yamaguchi et al. (46) \\
\hline \multicolumn{5}{|c|}{ Related to stress } \\
\hline Years & Basic research & Ghrelin & Index & References \\
\hline 2011 & Urocortin 1-treated rats & $\uparrow$ & Food intake $\uparrow$ & Yakabi et al. (47) \\
\hline 2011 & Novelty stressed mice & $\uparrow$ & Food intake $\uparrow$ & Saegusa et al. (29) \\
\hline 2013 & Novelty stressed mice & $\uparrow$ & Food intake $\uparrow$ & Yamada et al. (30) \\
\hline 2014 & Urocortin 1-treated rats & $\uparrow$ & Food intake $\uparrow$ & Yakabi et al. (32) \\
\hline 2014 & Acute restrained stressed mice & signal $\uparrow$ & Gastric motility $\uparrow$ & Nahata et al. (48) \\
\hline 2015 & Urocortin 1-treated rats & $\uparrow$ & Gastric emptying $\uparrow$ & Harada et al. (49) \\
\hline 2020 & Novelty stressed mice & - & Food intake $\uparrow$ & Yamada et al. (50) \\
\hline Years & Clinical research & Ghrelin & Index & References \\
\hline 2011 & Esophageal cancer patients with chemotherapy & - & Nausea $\downarrow$ & Seike et al. (51) \\
\hline 2014 & Non-erosive reflux disease & - & MCS score $\uparrow$ & Tominaga et al. (2) \\
\hline
\end{tabular}

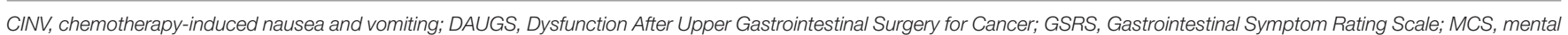
component summary.

rikkunshito contained a component with an antagonistic effect on the receptor $(32,49)$. However, the administration of an adrenergic $\beta 1$ receptor agonist also improves urocortin-induced ghrelin lowering, but it is not confirmed whether or not rikkunshito contains a $\beta 1$ agonist-like ingredients component.

Rodents are often bred and managed with 3-5 animals depending on the cage size. Acute stress can be induced by acclimating to this environment for about a week and then transferring to a completely new cage and bedding (2830, 50). Novel environmental changes can cause mild and transient corticosterone increase and decreased feeding in mice. Additionally, the concentration of acylated ghrelin in the blood decreases at the same time as stress loading (28-30). Rikkunshito significantly reduced the decrease in food intake and reduction in blood acylated ghrelin concentration due to this novel environmental change stress (28-30). Since the decreased food intake and ghrelin secretion in this model are partially canceled by the administration of $5-\mathrm{HT}_{2 \mathrm{~B}} \mathrm{R}$ or $5-\mathrm{HT}_{2} \mathrm{C}$ antagonists, serotonin is involved in the decreased food intake in the brain and digestive organs (28-30). ICV administration of CRF1R antagonists mediates reductions in food intake and plasma acylated ghrelin secretion (29), suggesting that intracerebral CRF1R activation is the trigger for the onset of this model. Restraint stress is known as classical physical and mental stress. Restraint stress in mice causes dysfunction of the upper gastrointestinal tract motility and a decrease in acylated/desacyl ghrelin ratio (48). Administration of rikkunshito to stress mice significantly improved gastric motor function abnormalities such as delayed gastric emptying and gastric motility index. It is speculated that these action by rikkunshito may have canceled the decreased feeding and ghrelin secretion deficiency due to stress.

\section{Clinical Study}

There are few clinical evidences focusing on the efficacy of rikkunshito on stress and mental illness. Tominaga et al. found that taking rikkunshito $(7.5 \mathrm{~g} /$ day $)$ for 8 weeks in patients with proton pump inhibitor-resistant non-erosive reflux disease (NERD) represents the mental quality of life in patients with a low body mass index. It proved that the mental component summary (MCS) scores of the SF-8 was significantly improved 
by rikkunshito (2). Additionally, administration of docetaxel/5FU/CDDP in patients with advanced esophageal cancer for 2 weeks with rikkunshito (7.5 g/day) significantly improved nausea, as well as sleep, mood, volition, daily living activity, and anxiety and greatly improved quality of life scores, including the feeling of anxiety (51). Although the results of these clinical trials do not show the direct anti-stress effect of rikkunshito, it led to the implementation of larger clinical trials and it is expected to find the usefulness of rikkunshito for gastrointestinal disorders and neuropsychiatric parameters due to stress loading.

\section{Mechanism of Action of Rikkunshito Antagonists on Serotonin, CRF, and Adrenergic Receptors}

Ghrelin release in the stomach and hypothalamus is negatively regulated by $5-\mathrm{HT}_{2 \mathrm{~B}} \mathrm{R}$ and ${ }_{2 \mathrm{C}} \mathrm{R}$ activation (11). Heptamethoxyflavone, hesperetin, nobiletin, tangeretin, and isoliquiritigenin, which are components of rikkunshito, have an antagonistic activity against $5-\mathrm{HT}_{2 \mathrm{~B}} \mathrm{R}$ and ${ }_{2 \mathrm{C}} \mathrm{R}$ in vitro. Additionally, these components, when administered alone in vivo, suppressed a decrease in blood ghrelin levels (11). Isoliquiritigenin has been confirmed to transfer to the brain after the administration of rikkunshito and may mediate the decrease in ghrelin secretion due to antagonism of $5-\mathrm{HT}_{2} \mathrm{C}$ localized in the central nervous system (56).

It is suggested that stress-related hypophagia involves abnormalities in ghrelin kinetics mediated by CRF1 and adrenergic receptors, and stimulation of CRF1 and $\alpha$ receptors negatively regulates ghrelin secretion. Nobiletin and isoliquiritigenin antagonize the CRF1 receptor at IC50 values of 0.36 and $0.67 \mu \mathrm{mol} / \mathrm{L}$, respectively (56). In addition, glycycoumarin has an IC50 value of 5-39 $\mu \mathrm{mol} / \mathrm{L}$ for all subtypes $(\mathrm{A}, \mathrm{B}, \mathrm{C})$ of the $\alpha_{2}$-adrenergic receptor (AR). The IC50 value of 6-shogaol, which is a component of Zingiberis rhizoma, is 25 $\mu \mathrm{mol} / \mathrm{L}$ for $\alpha_{2 \mathrm{~A}}-\mathrm{AR}, 8$-shogaol is $5-6 \mu \mathrm{mol} / \mathrm{L}$ for $\alpha_{2 \mathrm{~A}}, \alpha_{2 \mathrm{C}}-\mathrm{AR}$, and 10-gingerol is has an IC50 value of 5-31 $\mu \mathrm{mol} / \mathrm{L}$ for $\alpha_{2 \mathrm{~A}}$, $\alpha_{2 B}, \alpha_{2 C}-A R(32)$.

\section{Ghrelin Receptor Stimulating Effect}

Previous findings have shown that rikkunshito promotes acylated ghrelin secretion, but it does not increase it beyond physiological secretion. Therefore, it was questioned whether this degree of action could improve the reduced food intake. Rikkunshito enhanced the binding to ghrelin in cells expressing GHS-R1a in vitro and further significantly increased $\left[\mathrm{Ca}^{2+}\right]$ influx in the area under the curve by ghrelin. At the same time, it was discovered that the action was caused by the ingredients of rikkunshito, i.e., atractylodin (13). This finding means a new mechanism in which rikkunshito not only increases the blood acylated ghrelin concentration but also enhances the reactivity of acylated ghrelin with GHS-R1a, thereby increasing the ghrelin signal.

Another stimulating effect of the ghrelin signal has been studied and proposed. Rikkunshito mediates the production of cAMP through the inhibition of phosphodiesterase III against the adenylate cyclase-cAMP-PKA system involved in the ghrelin signal inhibitory effect of leptin via the PI3K-PDE pathway and the activation of ghrelin receptors (57). Further detailed research is required to determine how much this effect of rikkunshito affects the ghrelin signal.

\section{NEW POSSIBILITIES}

\section{Aging, Gender Difference, and Healthy Life Expectancy}

Maintaining the diet of the elderly is an important issue as a strategy to prevent sarcopenia in the global aging society. Additionally, aged people tend to lose their appetite due to changes in taste and decreased calorie consumption due to lack of exercise. Additionally, aged people often have many diseases, and maintaining the so-called healthy life expectancy and maintaining good quality of life are of utmost importance. In rodent studies, a flattening of ghrelin levels was observed in aged mice, with clearly reduced fasting ghrelin secretion compared to younger mice and, conversely, increased ghrelin levels during satiety (57). Additionally, changes in feeding behavior were also observed. Although the meal amount and meal size of the aged mice did not change compared with those of the younger mice, the number of activities at night and the food bout size were small, and the bout numbers were many. Thus, it eats little by little over time (58). Rikkunshito restores reduced feeding in aged mice without affecting acylated ghrelin levels (57).

So far, as a clinical evaluation, the impression that rikkunshito may be more effective for females than for males has been conveyed. However, there has been no evidence of gender differences in the effects of rikkunshito. Yamada et al. (50) studied and compared the effects of rikkunshito on decreased food intake in male and female mice exposed to psychological stress. Because of comparing female and male mice for events after stress loading, there was no gender difference in HPA axis activation, but food intake decreased more continuously in female mice. Female mice have a delayed increase in ghrelin rather than males and reduced responsiveness to exogenous ghrelin. In stress-loaded mice, rikkunshito showed an obvious effect of improving feeding in female mice. The cause of anorexia in female mice is thought to be ghrelin signal transduction failure in NTS, and rikkunshito was found to improve this signal disorder. Rikkunshito was more effective in women or the elderly (65 years and older) for FSSG in NERD patients (2). However, there is still no direct evidence that the action of rikkunshito as a ghrelin enhancer mediates gender differences and efficacy in the elderly. Further elucidation of the mechanism is required in the future.

It is known that calorie restriction activates various factors, including sirtuin (SIRT), and is involved in maintaining the functional decline of different tissues with aging (59). Particularly, activation of SIRT in the hypothalamus is triggered by calorie restriction and is associated with the maintenance of energy balance homeostasis (60-62). Ghrelin may increase the orexigenic signal and may cause the same condition as calorie restriction. For example, ghrelin activates adenosine monophosphate-activated protein kinase (AMPK) (63), and SIRT is also activated by AMPK (64). Therefore, it is easy to hypothesize that rikkunshito, which promotes ghrelin secretion 
and receptor activation, may cause SIRT activation. Fujitsuka et al. evaluated the effect of rikkunshito on healthy life expectancy using various aging-promoting mice (65). Rikkunshito induced activation of SIRT1 in the hypothalamus in vivo. It was also discovered that the effect was not expressed in ghrelin KO mice. Furthermore, administration of rikkunshito to ICR mice as aging-promoting models such as Klotho-deficient mice, SAMP8 mice, and normal-aged mice showed prolonged survival. Again, focal atrophy of myocardial fiber and pericarditis was significantly decreased in mice treated with rikkunshito. This study is a basic study using mice, but we hope that a clinical review will be conducted in the future and that rikkunshito will prove the possibility of improving the QOL of the elderly.

\section{SUMMARY}

The promoted ghrelin secretion and ghrelin signal promotion exerted by rikkunshito may play an important role for effectiveness for (i) anorexia, nausea and vomiting due to chemotherapy, (ii) severe loss of appetite and weight loss due to cancer cachexia and stress-induced hypophagia, and (iii) hypophagia in the elderly. Evidence accumulated over the last few years shows that rikkunshito is particularly effective against the loss of appetite and gastrointestinal disorders caused by chemotherapy. Additionally, as result of basic research,

\section{REFERENCES}

1. Miyoshi A, Taniuchi A, Masamune K, Masamune K, Ishikawa M, Fukutomi $\mathrm{H}$, et al. Clinical evaluation of Rikkunshito (TJ-43 TSUMURA \& Co) for indeterminate digestive complaints including chronic gastritis, etc Multi-institutional study with reference drug of Cisapride. Progress Med. (1991) 11:1605-31.

2. Tominaga K, Kato M, Takeda H, Shimoyama $\mathrm{Y}$, Umegaki E, Iwakiri $\mathrm{R}$, et al. A randomized, placebo-controlled, double-blind clinical trial of rikkunshito for patients with non-erosive reflux disease refractory to protonpump inhibitor: the G-PRIDE study. J Gastroenterol. (2014) 49:1392-405. doi: 10.1007/s00535-013-0896-9

3. Tominaga K, Sakata Y, Kusunoki H, Odaka T, Sakurai K, Kawamura O, et al. Rikkunshito simultaneously improves dyspepsia correlated with anxiety in patients with functional dyspepsia: a randomized clinical trial (the DREAM study). Neurogastroenterol Motil. (2018) 30:e13319. doi: 10.1111/nmo.13319

4. Kido T, Nakai Y, Kase Y, Sakakibara I, Nomura M, Takeda S, et al. Effects of rikkunshi-to, a traditional Japanese medicine, on the delay of gastric emptying induced by N(G)-nitro-L-arginine. J Pharmacol Sci. (2005) 98:1617. doi: 10.1254/jphs.FPJ04056X

5. Tatsuta M, Iishi H. Effect of treatment with liu-jun-zi-tang (TJ-43) on gastric emptying and gastrointestinal symptoms in dyspeptic patients. Aliment Pharmacol Ther. (1993) 7:459-62. doi: 10.1111/j.1365-2036.1993.tb00120.x

6. Kawahara H, Mitani Y, Nomura M, Nose K, Yoneda A, Hasegawa T, et al. Impact of rikkunshito, an herbal medicine, on delayed gastric emptying in profoundly handicapped patients. Pediatr Surg Int. (2009) 25:987-90. doi: 10.1007/s00383-009-2453-4

7. Mondal A, Takehara A, Aizawa S, Tanaka T, Fujitsuka N, Hattori $\mathrm{T}$, et al. Rikkunshito induces gastric relaxation via the beta-adrenergic pathway in Suncus murinus. Neurogastroenterol Motil. (2015) 27:875-84. doi: $10.1111 / \mathrm{nmo} .12564$

8. Kito Y, Suzuki H. Properties of Rikkunshi-to (TJ-43)-induced relaxation of rat gastric fundus smooth muscles. Am J Physiol rikkunshito may be involved in eating disorders and in extending healthy life expectancy in the elderly. However, based on the valuable basic research obtained so far, the evaluation of largescale clinical trials may lead to further evidence of the usefulness of rikkunshito for the benefit of patients, moreover even Japanese Kampo medicine.

\section{AUTHOR CONTRIBUTIONS}

CY and TH study design, data collection and analysis, and drafting of the manuscript. TH, SO, and HT study supervision. All authors contributed to the article and approved the submitted version.

\section{FUNDING}

This publication was funded by Tsumura \& Co.

\section{ACKNOWLEDGMENTS}

We would like to express our sincere gratitude to Prof. Yakabi, Prof. Uezono, and Prof. Inui and Asakawa for their guidance and support in basic research in this review. We would also like to thank Dr. Fujitsuka, Dr. Harada, Dr. Nahata, Dr. Mogami, and Dr. Koseki for their practical support for the research.
Gastrointestinal Liver Physiol. (2010) 298:G755-63. doi: 10.1152/ajpgi.0033 3.2009

9. Furukawa N, Manabe N, Kase Y, Hattori T, Imamura H, Kusunoki $\mathrm{H}$, et al. Intragastric infusion of rikkunshito (kampo) induces proximal stomach relaxation in conscious dogs. Auton Neurosci. (2013) 179:14-22. doi: 10.1016/j.autneu.2013.06.003

10. Shiratori M, Shoji T, Kanazawa M, Hongo M, Fukudo S. Effect of rikkunshito on gastric sensorimotor function under distention. Neurogastroenterol Motil. (2011) 23:323-9. doi: 10.1111/j.1365-2982.2010.01648.x

11. Takeda H, Sadakane C, Hattori T, Katsurada T, Ohkawara T, Nagai K, et al. Rikkunshito, an herbal medicine, suppresses cisplatin-induced anorexia in rats via 5-HT2 receptor antagonism. Gastroenterology. (2008) 134:2004-13. doi: 10.1053/j.gastro.2008.02.078

12. Yakabi K, Kurosawa S, Tamai M, Yuzurihara M, Nahata M, Ohno S, et al. Rikkunshito and 5-HT2C receptor antagonist improve cisplatin-induced anorexia via hypothalamic ghrelin interaction. Regul Pept. (2010) 161:97-105. doi: 10.1016/j.regpep.2010.02.003

13. Fujitsuka N, Asakawa A, Uezono Y, Minami K, Yamaguchi T, Niijima A, et al. Potentiation of ghrelin signaling attenuates cancer anorexia-cachexia and prolongs survival. Transl Psychiatry. (2011) 1:e23. doi: 10.1038/tp.2011.25

14. Kojima M, Hosoda H, Date Y, Nakazato M, Matsuo H, Kangawa K. Ghrelin is a growth-hormone-releasing acylated peptide from stomach. Nature. (1999) 402:656-60. doi: 10.1038/45230

15. Gutierrez JA, Solenberg PJ, Perkins DR, Willency JA, Knierman MD, Jin $\mathrm{Z}$, et al. Ghrelin octanoylation mediated by an orphan lipid transferase. Proc Natl Acad Sci U S A. (2008) 105:6320-5. doi: 10.1073/pnas. 0800708105

16. Schopfer LM, Lockridge O, Brimijoin S. Pure human butyrylcholinesterase hydrolyzes octanoyl ghrelin to desacyl ghrelin. Gen Comp Endocrinol. (2015) 224:61-8. doi: 10.1016/j.ygcen.2015.05.017

17. Hosoda H, Kangawa K. Standard sample collections for blood ghrelin measurements. Methods Enzymol. (2012) 514:113-26. doi: 10.1016/B978-0-12-381272-8.00008-8 
18. Gnanapavan S, Kola B, Bustin SA, Morris DG, McGee P, Fairclough P, et al. The tissue distribution of the mRNA of ghrelin and subtypes of its receptor, GHS-R, in humans. J Clin Endocrinol Metab. (2002) 87:2988. doi: 10.1210/jcem.87.6.8739

19. Stengel A, Goebel M, Wang L, Tache Y. Ghrelin, des-acyl ghrelin and nesfatin1 in gastric X/A-like cells: role as regulators of food intake and body weight. Peptides. (2010) 31:357-69. doi: 10.1016/j.peptides.2009.11.019

20. Cowley MA, Smith RG, Diano S, Tschop M, Pronchuk N, Grove KL, et al. The distribution and mechanism of action of ghrelin in the CNS demonstrates a novel hypothalamic circuit regulating energy homeostasis. Neuron. (2003) 37:649-61. doi: 10.1016/S0896-6273(03)00063-1

21. Fujimiya M, Itoh E, Kihara N, Yamamoto I, Fujimura M, Inui A. Neuropeptide $\mathrm{Y}$ induces fasted pattern of duodenal motility via $\mathrm{Y}(2)$ receptors in conscious fed rats. Am J Physiol Gastrointestinal Liver Physiol. (2000) 278:G32-8. doi: 10.1152/ajpgi.2000.278.1.G32

22. Fujino K, Inui A, Asakawa A, Kihara N, Fujimura M, Fujimiya M. Ghrelin induces fasted motor activity of the gastrointestinal tract in conscious fed rats. J Physiol. (2003) 550:227-40. doi: 10.1113/jphysiol.2003.040600

23. Zigman JM, Jones JE, Lee CE, Saper CB, Elmquist JK. Expression of ghrelin receptor mRNA in the rat and the mouse brain. J Comp Neurol. (2006) 494:528-48. doi: 10.1002/cne.20823

24. Diano S, Farr SA, Benoit SC, McNay EC, da Silva I, Horvath B, et al. Ghrelin controls hippocampal spine synapse density and memory performance. Nat Neurosci. (2006) 9:381-8. doi: 10.1038/nn1656

25. Fujitsuka N, Asakawa A, Hayashi M, Sameshima M, Amitani H, Kojima $\mathrm{S}$, et al. Selective serotonin reuptake inhibitors modify physiological gastrointestinal motor activities via 5-HT2c receptor and acyl ghrelin. Biol Psychiatry. (2009) 65:748-59. doi: 10.1016/j.biopsych.2008.10.031

26. Sakata I, Gong Z, Ikenoya C, Takemi S, Sakai T. The study of ghrelin secretion and acyl-modification using mice and ghrelinoma cell lines. Endocr J. (2017) 64:S27-S9. doi: 10.1507/endocrj.64.S27

27. Gupta D, Chuang JC, Mani BK, Shankar K, Rodriguez JA, OsborneLawrence $S$, et al. betal-adrenergic receptors mediate plasma acylghrelin elevation and depressive-like behavior induced by chronic psychosocial stress. Neuropsychopharmacology. (2019) 44:1319-27. doi: 10.1038/s41386-019-0334-7

28. Yamada C, Saegusa Y, Nakagawa K, Ohnishi S, Muto S, Nahata M, et al. Rikkunshito, a Japanese kampo medicine, ameliorates decreased feeding behavior via ghrelin and serotonin $2 \mathrm{~B}$ receptor signaling in a novelty stress murine model. Biomed Res Int. (2013) 2013:792940. doi: 10.1155/2013/792940

29. Saegusa Y, Takeda H, Muto S, Nakagawa K, Ohnishi S, Sadakane C, et al. Decreased plasma ghrelin contributes to anorexia following novelty stress. Am J Physiol Endocrinol Metab. (2011) 301:E685-96. doi: 10.1152/ajpendo.00121.2011

30. Yamada C, Sadakane C, Nahata M, Saegusa Y, Nakagawa K, Okubo N, et al. Serotonin $2 \mathrm{C}$ receptor contributes to gender differences in stressinduced hypophagia in aged mice. Psychoneuroendocrinology. (2015) 55C:8193. doi: 10.1016/j.psyneuen.2015.02.006

31. Zhao TJ, Sakata I, Li RL, Liang G, Richardson JA, Brown MS, et al. Ghrelin secretion stimulated by \{beta\}1-adrenergic receptors in cultured ghrelinoma cells and in fasted mice. Proc Natl Acad Sci U S A. (2010) 107:15868-73. doi: 10.1073/pnas.1011116107

32. Yakabi K, Harada Y, Takayama K, Ro S, Ochiai M, Iizuka S, et al. Peripheral alpha2-betal adrenergic interactions mediate the ghrelin response to brain urocortin 1 in rats. Psychoneuroendocrinology. (2014) 50:300-10. doi: 10.1016/j.psyneuen.2014.09.003

33. Matsumura T, Arai M, Yonemitsu Y, Maruoka D, Tanaka T, Suzuki T, et al. The traditional Japanese medicine Rikkunshito increases the plasma level of ghrelin in humans and mice. J Gastroenterol. (2010) 45:300-7. doi: 10.1007/s00535-009-0166-z

34. Yakabi K, Sadakane C, Noguchi M, Ohno S, Ro S, Chinen K, et al. Reduced ghrelin secretion in the hypothalamus of rats due to cisplatininduced anorexia. Endocrinology. (2010) 151:3773-82. doi: 10.1210/en.201 0-0061

35. Sadakane C, Muto S, Nakagawa K, Ohnishi S, Saegusa Y, Nahata M, et al. 10Gingerol, a component of rikkunshito, improves cisplatin-induced anorexia by inhibiting acylated ghrelin degradation. Biochem Biophys Res Commun. (2011) 412:506-11. doi: 10.1016/j.bbrc.2011.08.002
36. Yoshimura M, Matsuura T, Ohkubo J, Ohno M, Maruyama T, Ishikura $\mathrm{T}$, et al. The gene expression of the hypothalamic feeding-regulating peptides in cisplatin-induced anorexic rats. Peptides. (2013) 46:13-9. doi: 10.1016/j.peptides.2013.04.019

37. Taguchi M, Dezaki K, Koizumi M, Kurashina K, Hosoya Y, Lefor AK, et al. Total gastrectomy-induced reductions in food intake and weight are counteracted by rikkunshito by attenuating glucagon-like peptide-1 elevation in rats. Surgery. (2016) 159:1342-50. doi: 10.1016/j.surg.2015.12.003

38. Terawaki K, Kashiwase Y, Sawada Y, Hashimoto H, Yoshimura M, Ohbuchi $\mathrm{K}$, et al. Development of ghrelin resistance in a cancer cachexia rat model using human gastric cancer-derived 85As 2 cells and the palliative effects of the Kampo medicine rikkunshito on the model. PLoS ONE. (2017) 12:e0173113. doi: 10.1371/journal.pone.0173113

39. Ohno $\mathrm{T}$, Yanai M, Ando H, Toyomasu $\mathrm{Y}$, Ogawa A, Morita H, et al. Rikkunshito, a traditional Japanese medicine, suppresses cisplatininduced anorexia in humans. Clin Exp Gastroenterol. (2011) 4:291-6. doi: 10.2147/CEG.S26297

40. Takiguchi S, Hiura Y, Takahashi T, Kurokawa Y, Yamasaki M, Nakajima K, et al. Effect of rikkunshito, a Japanese herbal medicine, on gastrointestinal symptoms and ghrelin levels in gastric cancer patients after gastrectomy. Gastric Cancer. (2013) 16:167-74. doi: 10.1007/s10120-012-0164-3

41. Gunji S, Ueda S, Yoshida M, Kanai M, Terajima H, Takabayashi A. Effects of rikkunshito, a kampo medicine, on quality of life after proximal gastrectomy. J Surg Res. (2013) 185:575-80. doi: 10.1016/j.jss.2013.06.010

42. Ohnishi S, Watari H, Kanno M, Ohba Y, Takeuchi S, Miyaji T, et al. Additive effect of rikkunshito, an herbal medicine, on chemotherapy-induced nausea, vomiting, and anorexia in uterine cervical or corpus cancer patients treated with cisplatin and paclitaxel: results of a randomized phase II study (JORTC KMP-02). J Gynecol Oncol. (2017) 28:e44. doi: 10.3802/jgo.2017.28.e44

43. Harada T, Amano T, Ikari T, Takamura K, Ogi T, Fujikane T, et al. Rikkunshito for preventing chemotherapy-induced nausea and vomiting in lung cancer patients: results from 2 prospective, randomized phase 2 trials. Front Pharmacol. (2017) 8:972. doi: 10.3389/fphar.2017.00972

44. Yoshiya T, Mimae T, Ito M, Sasada S, Tsutani Y, Satoh K, et al. Prospective, randomized, cross-over pilot study of the effects of Rikkunshito, a Japanese traditional herbal medicine, on anorexia and plasma-acylated ghrelin levels in lung cancer patients undergoing cisplatin-based chemotherapy. Invest New Drugs. (2020) 38:485-92. doi: 10.1007/s10637-019-00836-X

45. Hamai Y, Yoshiya T, Hihara J, Emi M, Furukawa T, Yamakita I, et al. Traditional Japanese herbal medicine rikkunshito increases food intake and plasma acylated ghrelin levels in patients with esophageal cancer treated by cisplatin-based chemotherapy. J Thorac Dis. (2019) 11:2470-8. doi: $10.21037 /$ jtd.2019.05.67

46. Yamaguchi $H$, Kimura $Y$, Imamura $M$, Nagayama $M$, Ito $T$, Kyuno D, et al. Effect of rikkunshito, a traditional japanese herbal medicine, on delayed gastric emptying and oral dietary intake after pancreaticoduodenectomy: a prospective, randomized, single-center, openlabeled study. Clin Exp Gastroenterol. (2020) 13:577-87. doi: 10.2147/CEG. S252913

47. Yakabi K, Noguchi M, Ohno S, Ro S, Onouchi T, Ochiai M, et al. Urocortin 1 reduces food intake and ghrelin secretion via CRF(2) receptors. Am J Physiol Endocrinol Metab. (2011) 301:E72-82. doi: 10.1152/ajpendo. 00695.2010

48. Nahata M, Saegusa Y, Sadakane C, Yamada C, Nakagawa K, Okubo N, et al. Administration of exogenous acylated ghrelin or rikkunshito, an endogenous ghrelin enhancer, improves the decrease in postprandial gastric motility in an acute restraint stress mouse model. Neurogastroenterol Motil. (2014) 26:821-31. doi: 10.1111/nmo.12336

49. Harada Y, Ro S, Ochiai M, Hayashi K, Hosomi E, Fujitsuka N, et al. Ghrelin enhancer, rikkunshito, improves postprandial gastric motor dysfunction in an experimental stress model. Neurogastroenterol Motil. (2015) 27:1089-97. doi: $10.1111 /$ nmo. 12588

50. Yamada C, Iizuka S, Nahata M, Hattori T, Takeda H. Vulnerability to psychological stress-induced anorexia in female mice depends on blockade of ghrelin signal in nucleus tractus solitarius. Br J Pharmacol. (2020) 177:466682. doi: $10.1111 / \mathrm{bph} .15219$

51. Seike J, Sawada T, Kawakita N, Yamamoto Y, Yuasa Y, Yamai H, et al. A new candidate supporting drug, rikkunshito, for the QOL in advanced esophageal 
cancer patients with chemotherapy using Docetaxel/5-FU/CDDP. Int J Surg Oncol. (2011) 2011:715623. doi: 10.1155/2011/715623

52. Charmandari E, Tsigos C, Chrousos G. Endocrinology of the stress response. Annu Rev Physiol. (2005) 67:259-84. doi: 10.1146/annurev.physiol.67.040403.120816

53. Adam TC, Epel ES. Stress, eating and the reward system. Physiol Behav. (2007) 91:449-58. doi: 10.1016/j.physbeh.2007.04.011

54. Zellner DA, Loaiza S, Gonzalez Z, Pita J, Morales J, Pecora D, et al. Food selection changes under stress. Physiol Behav. (2006) 87:789-93. doi: 10.1016/j.physbeh.2006.01.014

55. Nahata M, Muto S, Nakagawa K, Ohnishi S, Sadakane C, Saegusa Y, et al. Serotonin $2 \mathrm{C}$ receptor antagonism ameliorates novelty-induced hypophagia in aged mice. Psychoneuroendocrinology. (2013) 38:2051-64. doi: 10.1016/j.psyneuen.2013.03.014

56. Mogami S, Sadakane C, Nahata M, Mizuhara Y, Yamada C, Hattori T, et al. CRF receptor 1 antagonism and brain distribution of active components contribute to the ameliorative effect of rikkunshito on stress-induced anorexia. Sci Rep. (2016) 6:27516. doi: 10.1038/srep27516

57. Takeda H, Muto S, Hattori T, Sadakane C, Tsuchiya K, Katsurada T, et al. Rikkunshito ameliorates the aging-associated decrease in ghrelin receptor reactivity via phosphodiesterase III inhibition. Endocrinology. (2010) 151:24452. doi: 10.1210/en.2009-0633

58. Yamada C, Mogami S, Hattori T. Psychological stress exposure to aged mice causes abnormal feeding patterns with changes in the bout number. Aging. (2017) 9:2269. doi: 10.18632/aging.101320

59. Maxmen A. Calorie restriction falters in the long run. Nature. (2012) 488:569. doi: $10.1038 / 488569$ a

60. Baur JA, Ungvari Z, Minor RK, Le Couteur DG, de Cabo R. Are sirtuins viable targets for improving healthspan and lifespan? Nat Rev Drug Discov. (2012) 11:443-61. doi: 10.1038/nrd3738

61. Toorie AM, Nillni EA. Minireview: Central Sirt1 regulates energy balance via the melanocortin system and alternate pathways. Mol Endocrinol. (2014) 28:1423-34. doi: 10.1210/me.2014-1115
62. Satoh A, Imai S. Hypothalamic Sirtl in aging. Aging. (2014) 6:1-2. doi: 10.18632/aging.100627

63. Kohno D, Sone H, Minokoshi Y, Yada T. Ghrelin raises [Ca2+]i via AMPK in hypothalamic arcuate nucleus NPY neurons. Biochem Biophys Res Commun. (2008) 366:388-92. doi: 10.1016/j.bbrc.2007.11.166

64. Canto C, Gerhart-Hines Z, Feige JN, Lagouge M, Noriega L, Milne JC, et al. AMPK regulates energy expenditure by modulating NAD+ metabolism and SIRT1 activity. Nature. (2009) 458:1056-60. doi: 10.1038/nature 07813

65. Fujitsuka N, Asakawa A, Morinaga A, Amitani MS, Amitani H, Katsuura G, et al. Increased ghrelin signaling prolongs survival in mouse models of human aging through activation of sirtuin1. Mol Psychiatry. (2016) 21:1613-23. doi: $10.1038 / \mathrm{mp} .2015 .220$

Conflict of Interest: $\mathrm{CY}$ and $\mathrm{TH}$ are employees of Tsumura \& Co. and get a salary from it. SO and HT receive research funds from Tsumura \& Co. The authors declare that this study received funding from Tsumura \& Co. The funder had the following involvement with the study: the decision to submit it for publication.

Publisher's Note: All claims expressed in this article are solely those of the authors and do not necessarily represent those of their affiliated organizations, or those of the publisher, the editors and the reviewers. Any product that may be evaluated in this article, or claim that may be made by its manufacturer, is not guaranteed or endorsed by the publisher.

Copyright $\odot 2021$ Yamada, Hattori, Ohnishi and Takeda. This is an open-access article distributed under the terms of the Creative Commons Attribution License (CC $B Y)$. The use, distribution or reproduction in other forums is permitted, provided the original author(s) and the copyright owner(s) are credited and that the original publication in this journal is cited, in accordance with accepted academic practice. No use, distribution or reproduction is permitted which does not comply with these terms. 